Z. Klin. Chem. Klin. Biochem.

10. Jg. 1972, S. $416-419$

\title{
Ammoniakverwertung und Harnstoffsynthese der isoliert perfundierten Rattenleber
}

\author{
Von H.-J. Mrertsch und L. Róka \\ Aus dem Institut für Klinische Chemie (Leiter: Prof. Dr. L. Róka) \\ der Justus Liebig-Universität Gießen
}

(Eingegangen am 2. Februar/17. Juli 1972)

Die isoliert perfundierte Rattenleber produziert bei der Perfusion mit einem erythrocytenfreien Medium, das als Sauerstoftträger eine Emulsion des Fluorkohlenwasserstoffes FC 75 enthält, maximal $2,1 \mu \mathrm{mol} \mathrm{Harnstoff} / \mathrm{h} \cdot \mathrm{g}$ Leber, solange kein geeignetes stickstoffhaltiges Substrat mit dem Perfusat angeboten wird. Bietet man mit dem Perfusat Ammoniumionen an, dann richtet sich die Harnstoffsynthese nach der angebotenen Ammoniumionenkonzentration bis zu einer Leistung von $34 \mu \mathrm{mol} \mathrm{Harnstoff} / \mathrm{h} \cdot \mathrm{g}$ Leber. Bei Angebot von physiologischen Ammoniumionenkonzentrationen ist das molare Verhältnis eliminiertes Ammoniak: synthetisiertem Harnstoff $=1$, bei Belastung der Leber mit höheren Ammoniumionenkonzentration steigt dieser molare Quotient bis nahezu 2.

\section{The utilization of ammonia and the synthesis of urea in the isolated perfused rat liver}

Isolated rat liver was perfused with an erythrocyte-free medium containing an emulsion of the fluorohydrocarbon FC 75 as an oxygen carrier. In the absence of a suitable nitrogen source a maximum of $2.1 \mu \mathrm{mol}$ urea were produced per $\mathrm{g}$ liver per hour. When ammonium ions were present in the perfusate, urea production increased, depending on the concentration of ammonium ions, to a maximum of $34 \mu \mathrm{mol}$ urea per $\mathrm{g}$ liver per hour. At physiological concentrations of ammonium ions the molar ratio of ammonium ions to synthesized urea is 1 . At higher concentrations of ammonium ions, this quotient increases to nearly 2.

Die isoliert perfundierte Rattenleber wird seit MrLLer (1) und Schrmassex $(2,3)$ als brauchbares Modell für das Studium verschiedener Leberfunktionen benutzt.

Die vorliegende Arbeit soll zeigen, ob sich an der isoliert perfundierten Rattenleber die Verwertung von exogen angebotenem Ammoniak für die Harnstoffsynthese untersuchen läßt.

Während der 3-stündigen Perfusion haben wir dazu die Elimination von Ammoniumionen aus dem Perfusat und die Abgabe von Harnstoff an das Perfusat gemessen. Die Ergebnisse zeigen, daß die Harnstoffsynthese der isoliert perfundierten Rattenleber von der jeweils angebotenen Ammoniakmenge abhängt.

\section{Material und Methodik}

Wir verwendeten normal ernährte Wistar-Ratten im Gewicht von $180-260 \mathrm{~g}$. Die Entnahme der Leber und die anschließende Perfusion der Leber erfolgte nach dem von Miller (1) und SchimasSEK (2) angegebenen Verfahren. Als Perfusionsmittel benutzten wir $100 \mathrm{ml}$ Krebs-Henselert-Puffer, in dem $5 \mathrm{~g}$ Rinderserumalbumin sowie $26 \mathrm{mg}$ Glucose und $50 \mu \mathrm{l} 5 \mathrm{proz}$. Bromthalein gelöst war. Als Sauerstoffträger wurde in diese Lösung $3 \mathrm{ml}$ des Fluorkohlenwasserstoffs FC $7^{1}$ ) durch Ultraschall einemulgiert, so $\mathrm{da} ß$ eine stabile Suspension entsteht mit Partikeln unter $5 \mu \mathrm{m}$ Größe (4). Das Perfusat wurde ständig mit $\mathrm{O}_{2} / \mathrm{CO}_{2}=95: 5(\nabla / v)$ gesättigt. Vor und während der Perfusion wurden in Abständen von $30 \mathrm{~min}$ Proben zur Analyse entnommen. Während der Perfusion wurde Ammoniumchlorid in Mengen zwischen 7,7 und $130 \mu \mathrm{mol} / \mathrm{h} \cdot \mathrm{g}$ Leber in Einzelportionen oder durch kontinuierliche Infusion zugegeben.

Harnstoff wird zum Teil mit den Testreagenzien der Firma Boehringer nach der Urease/BERTHELOT-Reaktion bestimmt, wobei jeweils die Berthelot-Reaktion ohne Urease als Leerwert be1) 3M-Company, Düsseldorf. nutzt wurde; in den meisten Versuchsieihen wurde Harnstoff mit der Diacetylmonoximmethode am Autoanalyzer bestimmt.

Ammoniak bestimmten wir enzymatisch nach der Methode von SCHMrdT und SCHWARZ (5).

Während der ganzen Versuchsreihe haben wir die Präzision von Tag zu Tag anhand von Kontrollseren kontrolliert. Wir verwendeten hintereinander drei verschiedene selbst hergestellte Serumpools. Mittelwert und Streuung betrugen:

Pool I $3488 \pm 254 \mu \mathrm{g} / 1$

Pool II $2351 \pm .81 \mu \mathrm{g} / 1$

Pool III $2971 \pm 255 \mu \mathrm{g} / \mathrm{l}$

Der Variationskoeffizient liegt damit im Verlauf der gesamten drei Versuchsperioden $z$ wischen 3,44 und $8,58 \%$ und etreicht nicht den von ENGlhardT (6) für die Phenol-HypochloritMethode angegebenen Wert von 2,71\%.

Die Zuverlässigkeit der Ammoniakbestimmung wurde darüberhinaus an einer Blindperfusion; bei der keine Leber in der Perfusionsapparatur eingeschaltet war, geprüft. In den im Abstand wie bei einer Perfusion entnommenen Proben wurde bei einer durch Infusor kontinuierlich zugeführten Ammoniakmenge von $112 \mu \mathrm{mol}$ Ammoniumchlorid/h 92 bis $93 \%$ wiedergefunden.

Glucose bestimmen wir mit einer Glucoseoxidase/Perid-Methode der Firma Boehringer (7), Bromthalein im Perfusat und der Galle photometrisch.

Die Bromthaleinclearance wurde aus der Abnahme der Bromthaleinkonzentration im Perfusat berechnet.

Kalium wurde flammenphotometrisch bestimmt;

die Enzymaktivitäten Lactatdehydrogenase (8), Alanin- (9) und Aspartattransaminase (10), sowie Glutamatdehydrogenase (11) mit den Reagenzien der Firma Boehringer.

Die mathematische Behandlung der Ergebnisse erfolgte nach den statistischen Verfahren der Varianzanalyse (partiell hierarchisches Modell)2).

2) Wir danken dem Institut für Medizinische Statistik und Dokumentation Gießen, (Leiter: Prof. Dr. J. DuDECK) für die Hilfe und Durchführung der statistischen Berechnung. 


\section{Ergebnisse}

Belastung der gesunden Leber mit Ammoniak Geht man davon aus, daB die Normalproduktion von Harnstoff in $24 \mathrm{~h}$ bei einem gesunden Menschen von $70 \mathrm{~kg} 30 \mathrm{~g}$ beträgt, dann würden sich daraus für eine $200 \mathrm{~g}$ schwere Ratte eine Harnstoffproduktion von $60 \mu \mathrm{mol} \mathrm{Harnstoff/h}$ errechnen. Setzt man als Stickstoffquelle pro mol Harnstoff 2 mol Ammoniak ein, dann folgert, daß die Leber einer $200 \mathrm{~g}$ schweren Ratte pro Stunde $120 \mu \mathrm{mol}$ Ammoniak verwerten kann.

Wir haben in unseren Versuchen die perfundierte Leber mit Dosen bis zu $9350 \mu \mathrm{mol}$ Ammoniak belastet. Bei einem Durchschnittsgewicht der Leber von $8 \mathrm{~g}$ entspricht das $390 \mu \mathrm{mol} / \mathrm{h} \cdot \mathrm{g}$ Leber.

Die Elimination von Ammoniumionen aus dem Perfusat haben wir in den beiden Versuchsreihen mit dem Angebot von 280 und $112 \mu \mathrm{mol}$ Ammoniumionen pro $h$ verfolgt.

Bei einer Zufuhr von $14 \mu \mathrm{mol} / \mathrm{h} \cdot \mathrm{g}$ Leber verwertet die Leber den allergrößten Teil, nämlich stündlich 13,5 $\mu \mathrm{mol}$ und der Ammoniakspiegel im Perfusat kann zwischen 105 und $165 \mu \mathrm{mol} / 1$ gehalten werden. Bei dem Angebot von $35 \mu \mathrm{mol}$ Ammoniak/h $\cdot \mathrm{g}$ Leber steigt der von der Leber verwertete Anteil auf rund $33,7 \mu \mathrm{mol} / \mathrm{h}$ an. Der nicht verwertete Rest kumuliert jedoch im Perfusat, so daß nach $3 \mathrm{~h}$ sich bereits ein Ammoniakspiegel von $475 \mu \mathrm{mol} / 1$ eingestellt hat (Abb. 1).

Die gesunde Leber kann sich an ein vermehrtes Ammoniakangebot anpassen, jedoch unter Verlust der Regulationsfähigkeit des Ammoniakspiegels im Perfusat. Offensichtlich wird durch Erhöhung des extrazellulären Ammoniakspiegels die Ammoniakaufnahme

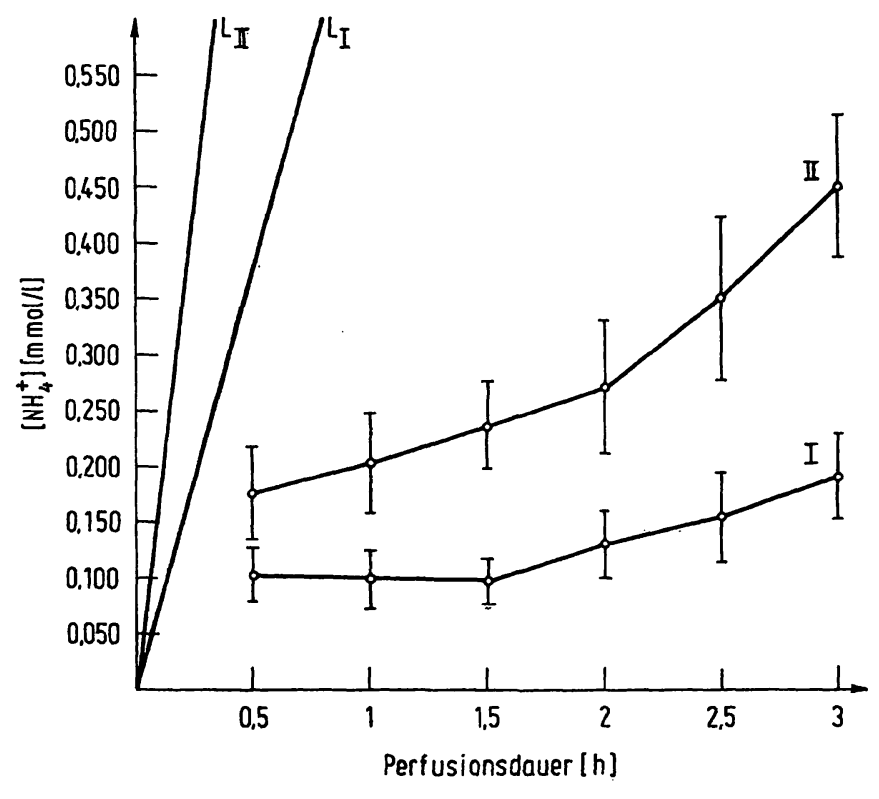

Abb. 1

Anstieg der Ammoniakkonzentration im Perfusat bei verschieden hoher Ammoniakzufuhr:

1.: $14 \mu \mathrm{mol} / \mathrm{h} \cdot \mathrm{g}$ Leber $(\mathrm{n}=10)$ Streuung $\pm 1 \mathrm{~s}$

li.: $35 \mu \mathrm{mol} / \mathrm{h} \cdot \mathrm{g}$ Leber $(\mathrm{n}=10)$ Streunng $\pm 1 \mathrm{~s}$

Der Unterschied ist signifikant $p<0,01$. Die Geraden $L_{I}$ und $L_{I}$ zeigen den Anstieg der Ammoniakkonzentration im Perfusionsleer versuch

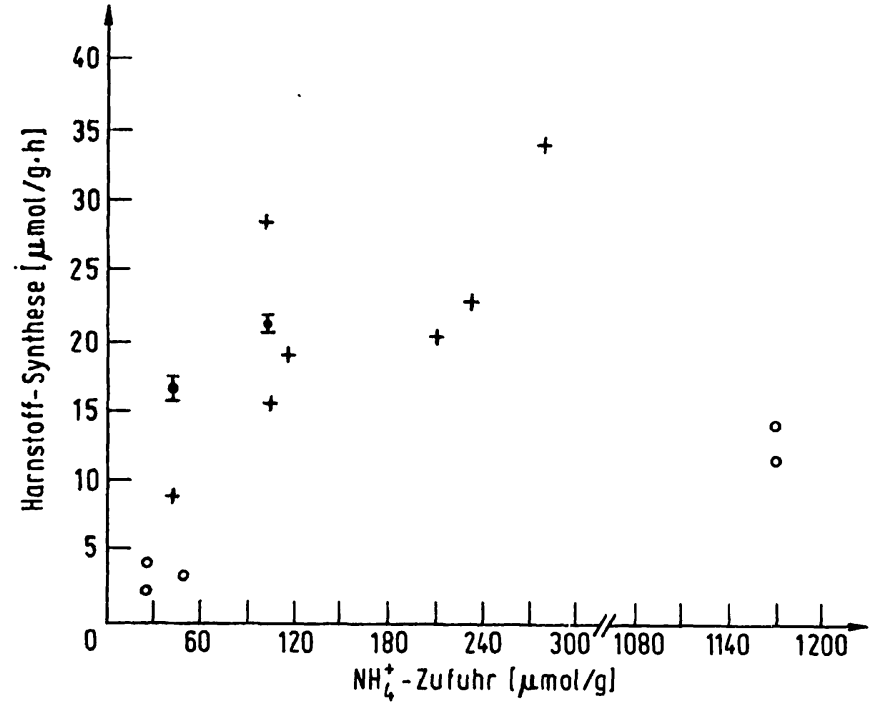

Abb. 2

Von cer Leber synthetisierte Mengen Harnstoff $[\mu \mathrm{mol} / \mathrm{h} \cdot \mathrm{g}$ Leber] in Abhängigkeit von der angebotenen Menge $\mathrm{NH}_{\ddagger}^{+}$bei unterschiedlicher Zufuhr

- Einmaliger Zusatz der Gesamtmenge zu Beginn der Perfusion - Kontinuierlich mit Infusionspumpe einschließlich Streubereich $\pm s$

in die Leberzelle begünstigt. Parallel dazu ist zu erwarten, daß auch die Harnstoffsynthese pro Zeiteinheit entsprechend ansteigt.

\section{Harnstoffsynthese}

Die Harnstoffsynthese in der perfundierten isolierten Rattenleber haben wir dadurch bestimmt, daß wir gemessen haben, wieviel Harnstoff von der Leber an das Perfusat abgegeben wird. Ohne exogenes Angebot von Ammoniumionen produziert die gesunde perfundierte Rattenleber 0 bis $2,1(\overrightarrow{\mathrm{x}}=0,709 ; \mathrm{n}=10) \mu \mathrm{mol}$ Harnstoff/h $\cdot \mathrm{g}$ Leber.

Bietet man der Leber Ammoniumionen mit dem Perfusat an, dann steigt die Harnstoffsynthese mit der angebotenen Ammoniumionenmenge bis $\mathrm{zu}$ einer Leistung von etwa $34 \mu \mathrm{mol} \mathrm{Harnstoff/h} \cdot \mathrm{g}$ Leber. Bei dem extrem hohen Wert eines einmaligen Angebotes von $1170 \mu \mathrm{mol}$ Ammoniumionen/g Leber lagen die synthetisierten Harnstoffmengen mit 12 bis $14 \mu \mathrm{mol} /$ $\mathrm{h} \cdot \mathrm{g}$ Leber deutlich unter der normalen Kapazität (Abb. 2).

Tabelle 1 zeigt die Ergebnisse bei den Fällen, bei denèn wir sowohl die Abnahme des Ammoniakspiegels wie auch die Synthese von Harnstoff jeweils parallel während der Versuchsdauer gemessen haben.

Die beiden Kurven (Abb. 3) sind Mittelwerte aus je 10 Versuchen. Man erkennt, daß die Harnstoffproduktion zunimmt, wenn mehr Ammoniak angeboten wird. In Abbildung 4 ist die jeweils gebildete Harnstoffmenge zu der verbrauchten Ammoniakmenge in Relation gestellt. Die Werte der beiden Versuchsgruppen liegen auf verschiedenen Graden. Errechnet man daraus den molaren Quotienten Ammoniak: Harnstoff, dann ergibt sich für die Versuchsgruppen mit $14 \mu \mathrm{mol} \mathrm{Ammoniak/h \cdot g} \mathrm{Leber} \mathrm{ein} \mathrm{Quotient} \mathrm{von}$ rund 1, während die Gruppe mit $35 \mu \mathrm{mol}$ Ammonial/ 
Tab. 1

Parallelbestimmungen von Ammoniakverwertung und Harnstoffsynthese

\begin{tabular}{|c|c|c|c|c|c|c|c|}
\hline \multirow{2}{*}{\multicolumn{2}{|c|}{$\mu \mathrm{mol} \mathrm{NH}+\mathrm{h} \cdot \mathrm{g}$ Leber }} & \multirow{2}{*}{\multicolumn{3}{|c|}{ Harnstoffsynthese $\mu \mathrm{mol} / \mathrm{h} \cdot \mathrm{g}$ Leber }} & \multirow{2}{*}{\multicolumn{3}{|c|}{$\frac{\text { mol NHt-Verwertung }}{\text { mol Harnstoft-Synthese }}$}} \\
\hline & & & & & & & \\
\hline angeboten & verwertet & $1 \mathrm{~h}$ & $2 \mathrm{~h}$ & $3 \mathrm{~h}$ & $1 \mathrm{~h}$ & $2 \mathrm{~h}$ & $3 \mathrm{~h}$ \\
\hline $\begin{array}{l}14,1(n=10) \\
35,1(n=10)\end{array}$ & $\begin{array}{l}13,4 \\
33,4\end{array}$ & $\begin{array}{l}19,2 \pm 1,1 \\
19,2 \pm 0,8\end{array}$ & $\begin{array}{l}13,1 \pm 1,5 \\
26,1 \pm 2,9\end{array}$ & $\begin{array}{l}13,9 \pm 2,6 \\
18,2 \pm 2,6\end{array}$ & $\begin{array}{r}0,68 \\
1,73\end{array}$ & $\begin{array}{l}1,03 \\
1,31\end{array}$ & $\begin{array}{l}0,97 \\
1,77\end{array}$ \\
\hline
\end{tabular}

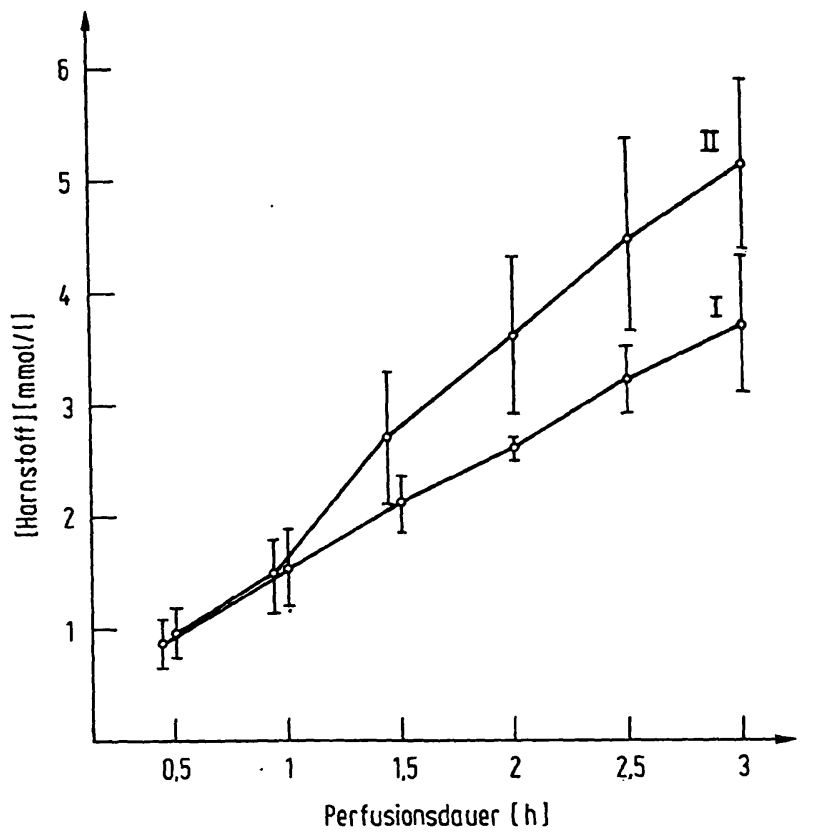

Abb. 3

Verlauf der Harnstoffkonzentration im Perfusionsmedium bei verschieden hoher Ammoniakzufuhr

I.: $14 \mu \mathrm{mol} / \mathrm{h} \cdot \mathrm{g}$ Leber $(\mathrm{n}=10)$ Streuung $\pm 1 \mathrm{~s}$

II.: $35 \mu \mathrm{mol} / \mathrm{h} \cdot \mathrm{g}$ Leber $(n=10)$ Streuung $\pm 1 \mathrm{~s}$ $35 \mu \mathrm{mol} / \mathrm{h} \cdot \mathrm{g}$ Leber $(\mathrm{n}=10)$ Streuung
Der Unterschied ist signifikant $\mathrm{p}<0,01$

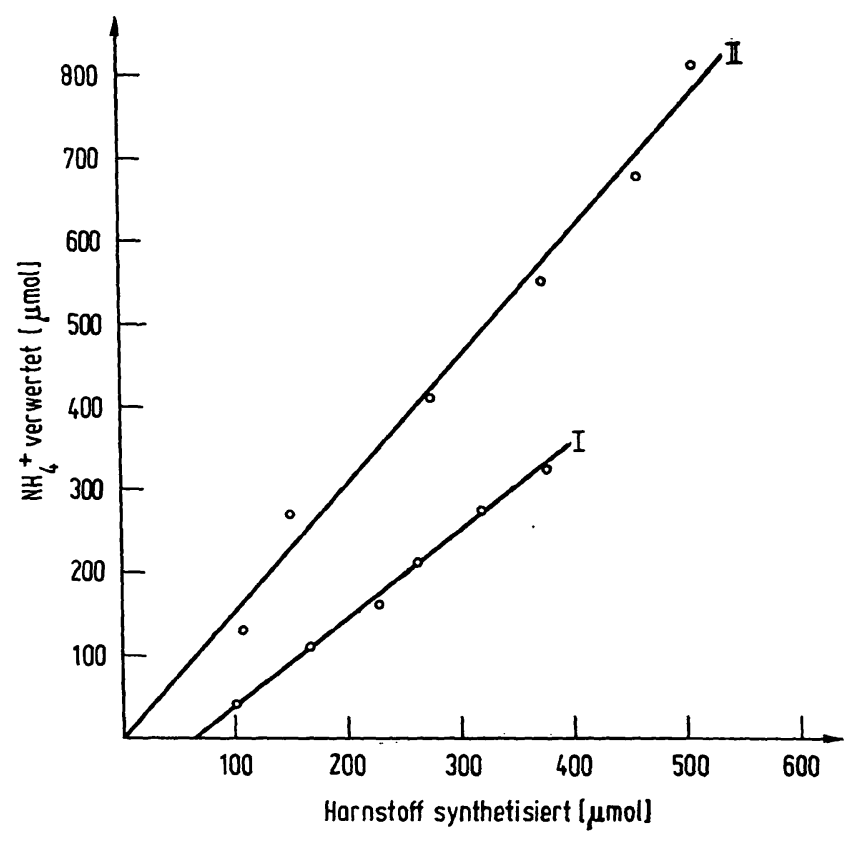

Abb. 4

Relation von verwertetem Ammoniak zu gebildetem Harnstoff bei den beiden Versuchsgruppen

I.: $14 \mu \mathrm{mol} / \mathrm{h} \cdot \mathrm{g}$ Leber $(\mathrm{n}=10)$
II.: $34 \mu \mathrm{mol} / \mathrm{h} \cdot \mathrm{g}$ Leber $(\mathrm{n}=10)$

Angegeben sind die zu verschiedenen Zeiten der Perfusion von der Gesamtleber verwertete $\mu$ mole Ammoniak und die im selben Zeitraum an das Perfusat abgegebenen $\mu$ mole Harnstoff $\mathrm{h} \cdot \mathrm{g}$ Leber einen Wert von nahezu 2 (s. Tab. 1) liefert.

Offenbar ändert sich etwas im Synthesemechanismus, wenn zu große Mengen Ammoniak pro Zeiteinheit in die Leber einströmen.

\section{Diskussion}

Die Harnstoffproduktion der isoliert perfundierten Rattenleber wurde von verschiedenen Untersuchern mitgeteilt (12-16). Ohne Zufuhr von stickstoffhaltigen Substanzen fand Mrrler $(12,13)$ eine Produktion von $6,4 \pm 1,6$ und Wirland (14) $7,8 \pm 1,2 \mu \mathrm{mol}$ Harnstoff/h, $\mathrm{g}$ Leber. Bei unseren Vèrsuchen lag die endogene Harnstoffproduktion bei maximal 2,1 $\mu \mathrm{mol}$ Harnstoff/h $\cdot g$ Leber.

Möglicherweise besteht der Unterschied in der anderen Versuchsanordnung. MTLLER und WreLAND benutzten als Sauerstoffträger Erythrocyten, wir FC 75 (17).

In den Harnstoffzyklus mündet 1 Molekül Ammoniak in Form von Carbamylphosphat. Die zweite Amidgruppe des Harnstoffmoleküls stammt aus der Aminogruppe eines Moleküls Asparaginsäure. Nimmt man an, da $B$ die gesunde Leber aus ihrem Pool ausreichende Mengen Asparaginsäure für den Harnstoffzyklus zur Verfügung stellen kann, dann wird pro synthetisiertem Molekül Harnstoff 1 Molekül Ammoniak verwertet, so daß der molare Quotient Ammoniak: Harnstoff $=1$ beträgt: Bei einem Uberangebot von Ammoniak werden jedoch offenbar 2 Moleküle Ammoniak pro synthetisiertem Harnstoff in der Leber fixiert. Aufgrund

Tab. 2

Leberleistungen bei Zufuhr unphysiologisch hoher Dosen an Ammoniak. Die angegebenen Werte sind Mittelwerte aus jeweils 10 Versuchen. $\mathrm{BSP}=$ Bromthalein; $\mathbf{n} . \mathbf{s} .=$ nicht signifikant

\begin{tabular}{|c|c|c|c|}
\hline & \multicolumn{3}{|c|}{$\begin{array}{l}\text { Ammoniakzufuhr } \\
(\mu \mathrm{mol} / \mathrm{h} \cdot \mathrm{g} \text { Leber })\end{array}$} \\
\hline & 14 & & Signifikanz \\
\hline Galleriproduktion $(\mu 1 / 3 \mathrm{~h})$ & 554 & 540 & \\
\hline BSP-Clearance HWZ (min) & 67 & 82 & \\
\hline BSP-Konzentration in der Galle/ & & & \\
\hline $\begin{array}{l}\text { Konzentration im Perfusat } \\
\text { Kalium }(\mu \mathrm{mol} / \mathrm{g} \text { Leber an Perfusat }\end{array}$ & 13,3 & 13,1 & \\
\hline $\begin{array}{l}\text { abgegeben) } \\
\text { Lactatdehydrogenase (mU/h } \cdot g \text { Leber }\end{array}$ & 1,5 & 0,5 & n.s. \\
\hline $\begin{array}{l}\text { an Perfusat abgegeben) } \\
\text { Alanintransaminase }(\mathrm{mU} / \mathrm{h} \cdot \mathrm{g} \text { Leber }\end{array}$ & 164,5 & 161,9 & n. s. \\
\hline $\begin{array}{l}\text { an Perfusat abgegeben) } \\
\text { Aspartattransaminase (mU/h } \cdot g \text { Leber }\end{array}$ & 15,6 & 13,5 & n.s. \\
\hline $\begin{array}{l}\text { an Perfusat abgegeben) } \\
\text { Glutamatdehydrogenase }(\mathrm{mU} / \mathrm{h} \cdot \mathrm{g}\end{array}$ & 91,6 & 81,8 & $p=0,01$ \\
\hline Leber an Perfusat abgegel & 2,0 & 1,9 & n. s. \\
\hline Glucose (mmol/l im Perfusat nach $3 \mathrm{~h}$ ) & $\mathbf{5 , 7}$ & 6,5 & n. s. \\
\hline
\end{tabular}


unserer Versuche können wir nicht entscheiden, ob beide Moleküle Ammoniak in den neu synthetisierten Harnstoff eingebaut werden oder ob das zweite Molekül Ammoniak in Form irgendeines Intermediärproduktes, z. B. als Glutaminsäure oder als Asparaginsäure in der Leber verwertet wird.

Aus Tabelle 2 erkennt man, daß auch bei Zufuhr von unphysiologisch hohen Dosen an Ammoniumionen die übrigen Leberleistungen, wie Glucoseregulationen, Gallenproduktion und Bromthaleinclearance erhalten bleiben. Verglichen mit Lebern, denen nur physiologische Dosen an Ammoniumionen angeboten wurden, bleibt auch der Austritt von $\mathrm{K}^{+}$sowie cytoplasmatischer (Lactatdehydrogenase, Alanintransaminase) und mitrochondrialer (Aspartattransaminase, Glutamatdehydrogenase) Enzyme unverändert.

\section{Literatur}

1. Mrller, L. L. \& Watson, M. L. (1951), J. Exp. Med. 94, 435-543. - 2. SchimasseK, H. (1963), Biochem. Z. 336, 460-467. - 3. Schrmassex, H. (1963), Biochem. Z. 336, 468-473. - 4. GeYer, R. P. (1970), Medizin u. Ernährung 11, 256-261. 5. Scharrdt, F. H. \& Schwarz, M. (1966), Klin. Wochenschr. 44, 591-592. - 6. Englhardt, A., Haertwig, B. \& Franken, F. (1971), diese Z. 9, 229-234. - 7. BerGMEYER, H. U. \& BERNT, E. (1970), in Methoden der enzymatischen Analyse (Hrsg. BerGMEYeR, H. U.), 2. Aufl., S. 1179-1180, Verlag Chemie. 8. Bergameyer, H. U. \& BerNT, E. (1970), in: 1. c. (7) S. 533. 9. Bergmeyer, H. U. \& BerNT, E., in: I. c. (7) S. 717. - 10.
Bergmeyer, H. U. \& Bernt, E., in: 1. c. (7) S. 685. - 11. SchmidT, E., in: 1. c. (7) S. 607. - 12. MILler, L. L. (1965), Fed. Proc. Fed. Amer. Soc. Exp. Biol. 24, 737-744. - 13. Miller, L. L. (1960), Nature (London) 185, 248. - 14. Menahan, L. A. \& WIELAND, O. (1969), Eur. J. Biochem. 9, 55-62. 15. Söling, N. D., Willms, B., Janson, G. \& Mitrenga, A. (1970), in: Recent Hypolgycemic Sulfonylurea Derivatives (Hrsg. H. D. Söling) S. 130 ff. Huber Verlag, Bern. - 16. Wal, TER, P., Piquerez, R. J. \& Anabitarto, M. (1970), in: 1. c. (15) S. 130 ff. 17. HaAs, H. \& Fetzer, S. (1971), Anatomischer Anzeiger 128, 319-320. -
Prof. Dr. L. Róka 6300 Gießen

Klinikstr. 32b 